\title{
Article
}

\section{Scattering of Matter Wave Solitons on Localized Potentials}

\author{
Sidse Damgaard Hansen, Nicolai Nygaard and Klaus Mølmer *
}

Department of Physics and Astronomy, University of Aarhus, Ny Munkegade 120, DK-8000 Aarhus C, Denmark; SIDHA@orsted.dk (S.D.H.); nicny@orsted.dk (N.N.)

* Correspondence: moelmer@phys.au.dk

\section{check for}

updates

Citation: Damgaard Hansen, S.; Nygaard, N.; Mølmer, K. Scattering of Matter Wave Solitons on Localized Potentials. Appl. Sci. 2021, 11, 2294. https://doi.org/10.3390/app11052294

Academic Editor: Chiara D’Errico

Received: 22 January 2021

Accepted: 18 February 2021

Published: 5 March 2021

Publisher's Note: MDPI stays neutral with regard to jurisdictional claims in published maps and institutional affiliations.

Copyright: (c) 2021 by the authors. Licensee MDPI, Basel, Switzerland. This article is an open access article distributed under the terms and conditions of the Creative Commons Attribution (CC BY) license (https:// creativecommons.org/licenses/by/ $4.0 /)$.

\begin{abstract}
We study the reflection and transmission properties of matter wave solitons impinging on localized scattering potentials in one spatial dimension. By mean field analysis we identify regimes where the solitons behave more like waves or more like particles as a result of the interplay between the dispersive wave propagation and the attractive interactions between the atoms. For a bright soliton propagating together with a dark soliton void in a two-species Bose-Einstein condensate, we find different reflection and transmission properties of the dark and the bright components.
\end{abstract}

Keywords: Bose-Einstein condensates; non-linear Schrödinger equation; solitons; scattering; collective excitations; atomtronics

PACS: 03.75.Lm; 05.45.Yv; 03.75.-b

\section{Introduction}

Solitons are exceptionally stable wave phenomena that appear in a variety of physical systems. They propagate without spreading due to a fine balance between the dispersion and the non-linearity of the physical system. After their discovery as stable waves in shallow waters by Russell in 1834, solitons have been found in very different non-linear systems. As an example, both bright optical solitons which are peaks in the intensity and dark solitons which are intensity minima have found applications in optical transmission through fibres [1,2]. In this article we consider soliton waves in Bose-Einstein condensates of neutral atoms. Laser fields may be employed to induce waveguides, potential barriers and filters and thus enable the matter wave functionalities needed in the evolving field of atomtronics [3]. In the present work we show that in addition to their favorable propagation behavior, matter wave solitons have remarkable scattering properties that may be observed in the atomtronics architectures.

The condensation of a cold gas of atoms permits a description of the many-body problem by a single condensate wave function order parameter, which is in turn described by the non-linear Gross-Pitaevskii wave equation [4]. The non-linearity in Bose-Einstein condensates arises from the repulsive or attractive interactions of the particles in the condensate. In equivalence with non-linear optics, dark and bright solitons are, respectively, localized dips and peaks in the Bose-Einstein condensate density. In addition to single dark and bright solitons a variety of composite solitons, for example dark-bright solitons, can exist in spinor condensates [5-7].

The dynamics of solitons, bright and dark as well as composite dark-bright solitons, in inhomogeneous condensates with slowly varying potentials has been investigated and is adequately described by a particle-like behavior [8-15]. When solitons are scattered on a localized potentials both particle- and wave-like behavior is observed [16-32]. In this article, we shall further elaborate the analysis of matter wave solitons in 1D-condensates, investigating their scattering properties on localized potentials. We note that there is a rich literature [33-37] on the dynamics of non-linear superpositions of solitons, the so-called breathers and of their remarkable scattering properties $[38,39]$, which we shall not address in this article. 
In Section 2, we introduce the Gross-Pitaevskii equation and a convenient mapping to dimensionless coordinates that will be used throughout the paper. In Section 3, we introduce the bright, the dark, and the composite dark-bright solitons, and we briefly review their properties. In Section 4, we show that scattering of bright solitons on a low and wide potential barrier can be well understood as the motion of a classical particle governed by an effective Lagrangian, extracted from the quantum problem, while the scattering on high and narrow barriers can be understood from the single particle quantum problem. In Section 5, we present results for the scattering of a dark-bright soliton on a potential felt only by the bright soliton atoms, and we show to what extent their scattering is transferred to the dark soliton. Section 6 concludes the paper.

\section{Mean Field Theory of Interacting Atoms}

We treat the Bose-Einstein condensate of atoms at zero temperature in a Hartree mean field theory, which neglects particles outside the condensate and treats short range interactions in the gas by an interaction potential which is proportional to the mean atomic density. We assume either a guided condensate with a high transversal excitation frequency [40] or the situation of a wide condensate with approximate translational invariance in the transversal directions. This yields the 1D timedependent Gross-Pitaesvkii equation (GPE)

$$
\begin{aligned}
i \hbar \frac{\partial \psi(x, t)}{\partial t} & =\left(-\frac{\hbar^{2}}{2 m} \frac{\partial^{2}}{\partial x^{2}}+V(x, t)\right. \\
& \left.+g|\psi(x, t)|^{2}\right) \psi(x, t),
\end{aligned}
$$

where the order parameter, the macroscopic wave function, is normalized to the total number of atoms

$$
\int d x|\psi(x, t)|^{2}=N
$$

so that $|\psi(x, t)|^{2}$ represents the particle density. The 1D interaction strength $g$ is a known function of the 3D low energy scattering properties and the transverse confinement of the atoms [4].

In an attractive BEC with $g<0$, (meta-)stable bright soliton solutions with a finite number of particles exist, while in a repulsive BEC with $g>0$, dark solitons may exist in the form of holes in the background density.

For numerical purposes it is advantageous to rewrite the GPE in a dimensionless form, and for a homogeneous condensate with repulsive interactions, the linear atomic density, $n_{0}$ and the healing length $\xi=\sqrt{\frac{\hbar^{2}}{m g n_{0}}}$ provide natural scale factors. We thus introduce dimensionless quantities for length, energy and time, labelled by a tilde

$$
x=\tilde{x} \xi, \quad E=\tilde{E} \frac{\hbar^{2}}{m \xi^{2}}=\tilde{E} n_{0} g, \quad t=\tilde{t} \frac{m \xi^{2}}{\hbar} .
$$

and obtain the equation

$$
i \frac{\partial \tilde{\psi}}{\partial \tilde{t}}=-\frac{1}{2} \frac{\partial^{2} \tilde{\psi}}{\partial \tilde{x}^{2}}+|\tilde{\psi}|^{2} \tilde{\psi}+\tilde{V} \tilde{\psi}
$$

where the dimensionless wave function

$$
\tilde{\psi}=\frac{\psi}{\sqrt{n_{0}}}
$$

is normalized to unit bulk density $|\tilde{\psi}|^{2}=1$ in the homogeneous part of the condensate. 
A condensate with attractive interactions has no natural length scale like the healing length, but for convenience in the following, we introduce an arbitrary length unit $l$, which may be chosen to fit a relevant experimental length scale, and we set

$$
x=\tilde{x} l, \quad E=\tilde{E} \frac{\hbar^{2}}{m l^{2}}, \quad t=\tilde{t} \frac{m l^{2}}{\hbar} .
$$

Inserting this in the GPE, Equation (1), and introducing the rescaled wave function, $|\tilde{\psi}|^{2}=|g| \frac{m l^{2}}{\hbar^{2}}|\psi|^{2}$, leads to the dimensionless GPE,

$$
i \frac{\partial \tilde{\psi}}{\partial \tilde{t}}=-\frac{1}{2} \frac{\partial^{2} \tilde{\psi}}{\partial \tilde{x}^{2}}-|\tilde{\psi}|^{2} \tilde{\psi}+\tilde{V} \tilde{\psi}
$$

The rescaled number of particles in the condensate is

$$
\tilde{N}=\int \mathrm{d} \tilde{x}|\tilde{\psi}|^{2}=N \frac{m l|g|}{\hbar^{2}} .
$$

Thus for given $N$, the choice of $\tilde{N}$ determines the length scale $l$ and sets the dimensions of the problem.

Due to the presence of the non-linear term it is in general necessary to solve the GrossPitaesvkii equation by numerical means. The numerical results presented in the following are based on propagation of the one-dimensional, dimensionless and non-homogeneous GPE using the Crank-Nicolson method. For brevity we will omit the tilde above dimensionless quantities throughout.

\section{Solitons}

In this section we recall the soliton solutions of the homogeneous Gross-Piaevskii equation.

\subsection{Dark Solitons}

Dark solitons are formed in repulsive BECs, governed by the homogeneous GPE, Equation (4), and fulfil the boundary condition $\lim _{x \rightarrow \pm \infty}|\psi(x)|^{2}=n_{0}$, where $n_{0}$ is the bulk value of the condensate density and also the dimensionless chemical potential. A dark soliton moving with velocity $v$ is described by

$$
\psi_{\mathrm{D}}(x, t)=\sqrt{n_{0}}\left[i \frac{v}{c}+\sqrt{1-\frac{v^{2}}{c^{2}}} \tanh \left(\frac{x-v t}{\xi_{v}}\right)\right] \mathrm{e}^{-i \mu t / \hbar},
$$

where $\xi_{v}=\xi / \sqrt{1-\frac{v^{2}}{c^{2}}}$, with $\xi$ the healing length and $c=\sqrt{\frac{n_{0} g}{m}}$ the speed of sound, both unity in our dimensionless units [4]. The dark soliton has a density dip around $x=v t$, and we will characterize the size of the soliton by the number of atoms, $N_{\mathrm{D}}$, missing compared to the homogeneous case,

$$
N_{\mathrm{D}}=\int \mathrm{d} x\left(n_{0}-\left|\psi_{\mathrm{D}}\right|^{2}\right)=2 n_{0} \xi \sqrt{1-\frac{v^{2}}{c^{2}}}
$$

The density depression moves in the opposite direction of the atoms in the condensate, whose motion is given by the gradient of the phase of the condensate wave function. The energy of the dark soliton is given by the difference between the energy of the condensate containing a dark soliton and the energy of the homogeneous condensate,

$$
\begin{aligned}
E_{\mathrm{D}} & =\int \mathrm{d} x\left(\frac{\hbar^{2}}{2 m}\left|\frac{\partial \psi_{\mathrm{D}}}{\partial x}\right|^{2}+\frac{g}{2}\left(\left|\psi_{\mathrm{D}}\right|^{2}-n_{0}\right)^{2}\right) \\
& =\frac{4}{3} n_{0} \hbar c\left(1-\frac{v^{2}}{c^{2}}\right)^{3 / 2} .
\end{aligned}
$$


The soliton energy decreases with increasing soliton velocity, and for $\frac{v}{c} \ll 1$ one finds

$$
E_{\mathrm{D}} \simeq \frac{4}{3} n_{0} \hbar c-\frac{2 n_{0} \hbar}{c} v^{2}
$$

corresponding to an effective negative mass of $-4 n_{0} \frac{\hbar}{c}$.

\subsection{Bright Solitons}

If the interactions in the BEC are attractive $(g<0)$ the homogeneous GPE supports bright soliton solutions,

$$
\psi_{\mathrm{B}}(x, t)=\sqrt{\frac{2 \mu}{g}} \operatorname{sech}\left(\frac{\sqrt{2 m|\mu|}}{\hbar}(x-v t)\right) \mathrm{e}^{i(m v x-\omega t) / \hbar},
$$

where $\omega=\frac{1}{2} m v^{2}+\mu, \mu$ being the chemical potential [4]. The bright soliton propagates with velocity $v$, and contrary to the dark soliton, its shape does not depend on its velocity, but only on the number of atoms in the condensate.

In the dimensionless coordinates, the soliton solution, Equation (13), is

$$
\psi_{\mathrm{B}}(x, t)=A \operatorname{sech}[B(x-v t)] \mathrm{e}^{i(v x-\omega t)},
$$

where $A=B=N / 2$, the chemical potential of the condensate is $\mu=-\frac{B^{2}}{2}$, and $\omega=$ $\frac{v^{2}}{2}-\frac{B^{2}}{2}$.

The energy of the bright soliton, in the absence of an external potential is given by three contributions [19]:

$$
E=E_{\text {kin }}^{\mathrm{d}}+E_{\text {kin }}^{v}+E_{\text {int }} .
$$

The first part is the quantum pressure contribution to the kinetic energy

$$
E_{\text {kin }}^{\mathrm{d}}=\frac{1}{2} \int \mathrm{d} x\left|\frac{\partial|\psi|}{\partial x}\right|^{2}=\frac{N^{3}}{24} .
$$

This is present even when the soliton is stationary, while the contribution to kinetic energy from the phase gradient

$$
E_{\text {kin }}^{v}=\frac{1}{2} \int \mathrm{d} x|| \psi\left|\frac{\partial \mathrm{e}^{i(v x-\omega t)}}{\partial x}\right|^{2}=\frac{1}{2} N v^{2},
$$

disappears for a stationary soliton.

Finally, the interactions between the atoms contribute

$$
E_{\text {int }}=-\frac{1}{2} \int \mathrm{d} x|\psi|^{4}=-\frac{N^{3}}{12}
$$

to the energy of the soliton.

In total the energy of the bright soliton is

$$
E_{\mathrm{B}}=\frac{1}{2} N v^{2}-\frac{N^{3}}{24} .
$$

\subsection{Dark-Bright Solitons}

A dark-bright soliton is a composite object in a two-component condensate consisting of a bright soliton BEC and a density depression in a repulsive BEC. Here, we consider the case where all interactions, internal and between the two components, are repulsive with 
equal strength parameters in the coupled Gross-Pitaevskii Equations (note that this implies a factor two difference between the collision strengths of identical and different pairs atom)

$$
\begin{aligned}
i \frac{\partial \psi_{1}(x, t)}{\partial t}=( & -\frac{1}{2} \frac{\partial^{2}}{\partial x^{2}}+\left|\psi_{1}(x, t)\right|^{2} \\
& \left.+\left|\psi_{2}(x, t)\right|^{2}\right) \psi_{1}(x, t), \\
i \frac{\partial \psi_{2}(x, t)}{\partial t}=( & -\frac{1}{2} \frac{\partial^{2}}{\partial x^{2}}+\left|\psi_{2}(x, t)\right|^{2} \\
& \left.+\left|\psi_{1}(x, t)\right|^{2}\right) \psi_{2}(x, t),
\end{aligned}
$$

This non-linear, coupled set of differential equations has a dark-bright soliton solution:

$$
\begin{aligned}
\psi_{\mathrm{D}}(x, t)= & \left(i \sqrt{\mu} \sin \alpha+\sqrt{\mu} \cos \alpha \tanh \left[\kappa\left(x-x_{0}\right)\right]\right) \mathrm{e}^{-i \mu t}, \\
\psi_{\mathrm{B}}(x, t)= & \sqrt{\frac{N_{\mathrm{B}} \kappa}{2}} \mathrm{e}^{i x \kappa \tan \alpha} \operatorname{sech}\left[\kappa\left(x-x_{0}\right)\right] . \\
& \mathrm{e}^{-i\left(\frac{\kappa^{2} \tan ^{2} \alpha}{2}-\frac{\kappa^{2}}{2}+\mu\right) t},
\end{aligned}
$$

where

$$
\kappa^{2}+\frac{\kappa N_{\mathrm{B}}}{2}=\mu \cos ^{2} \alpha
$$

and

$$
x_{0}=v t
$$

with $v=\kappa \tan \alpha[5,41]$.

The bright soliton can only exists in the repulsive condensate because it is trapped inside the dark soliton. The larger the number of particles in the bright component, the wider is the dark-bright soliton due to the repulsive interaction between the two components.

The energy of the two-component condensate can be separated into three parts,

$$
E_{\mathrm{DB}}=E_{\mathrm{D}}+E_{\mathrm{B}}+E_{\mathrm{int}}
$$

where the energy of the dark soliton $E_{\mathrm{D}}$ is defined by subtracting the energy of the dark background condensate,

$$
\begin{aligned}
E_{\mathrm{D}} & =\int \mathrm{d} x\left(\frac{1}{2}\left|\frac{\partial \psi_{\mathrm{D}}}{\partial x}\right|^{2}+\frac{1}{2}\left(\left|\psi_{\mathrm{D}}\right|^{2}-\mu\right)^{2}\right) \\
& =\frac{2}{3} \kappa \mu \cos ^{2} \alpha+\frac{2}{3} \frac{\mu^{2} \cos ^{4} \alpha}{\kappa},
\end{aligned}
$$

$E_{\mathrm{B}}$ is the energy of the bright soliton

$$
\begin{aligned}
E_{\mathrm{B}} & =\int \mathrm{d} x\left(\frac{1}{2}\left|\frac{\partial \psi_{\mathrm{B}}}{\partial x}\right|^{2}+\frac{1}{2}\left|\psi_{\mathrm{B}}\right|^{4}\right) \\
& =\frac{1}{2} N_{\mathrm{B}} v^{2}+\frac{1}{6} N_{\mathrm{B}} \kappa\left(N_{\mathrm{B}}+\kappa\right),
\end{aligned}
$$


and $E_{\text {int }}$ is the energy caused by the interaction between the two components

$$
\begin{aligned}
E_{\text {int }} & =\int \mathrm{d} x\left|\psi_{\mathrm{B}}\right|^{2}\left|\psi_{\mathrm{D}}\right|^{2} \\
& =N_{\mathrm{B}} \mu \sin ^{2} \alpha+\frac{1}{3} N_{\mathrm{B}} \mu \cos ^{2} \alpha .
\end{aligned}
$$

The total vector soliton energy can then be written as [41]

$$
E_{\mathrm{DB}}=\frac{4}{3} \kappa^{3}+\frac{1}{2} N_{\mathrm{B}} \kappa^{2}+\frac{1}{2} N_{\mathrm{B}} v^{2}+\mu N_{\mathrm{B}} .
$$

\section{Scattering of Bright Solitons}

Bright solitons are localized objects and in weakly varying potentials where the nonlinearity is important, they largely behave as classical particles [16-18,26,42,43], whereas in strongly varying potentials we expect wave diffraction phenomena to enter [18-26,42-44]. In this Section, we shall investigate the transition between particle and wave behavior of bright solitons scattering on simple square potentials in one dimension.

\subsection{Weak Barriers}

As mentioned in the previous section, the chemical potential of a bright soliton is given by $\mu=-\frac{N^{2}}{8}$, and when the potential is weak compared to the chemical potential, $N^{2} \gg V_{0}$, we expect the strong interaction potential to hold the atoms together throughout the scattering process.

In Figure 1 the time evolution of the Gross-Pitaevskii wave function is shown for soliton wave packets, incident on a weak square barrier with four different incident velocities. The calculations show that the soliton keeps its sech-shape and is either reflected or transmitted by the barrier like a classical particle.

In Figure $1 b, c$ the soliton is slowed down dramatically and dwells on the potential for a rather long time, before it is eventually reflected or transmitted regaining its initial shape and speed.

We define reflection and transmission coefficients as the weight of the wave function on the left and right hand side of the barrier

$$
\begin{aligned}
& R=\frac{1}{N} \int_{-L / 2}^{0} \mathrm{~d} x|\psi|^{2} \\
& T=\frac{1}{N} \int_{0}^{L / 2} \mathrm{~d} x|\psi|^{2},
\end{aligned}
$$

where $L$ is the length of the box used for our calculations, and the barrier is centred at $x=0$. In Figure 2 transmission and reflection coefficients are shown for two different barrier widths. The figure confirms that the solitons are almost exclusively reflected or transmitted by the wide barrier, while the narrow barrier causes a minor splitting of the mean field wave packet. 


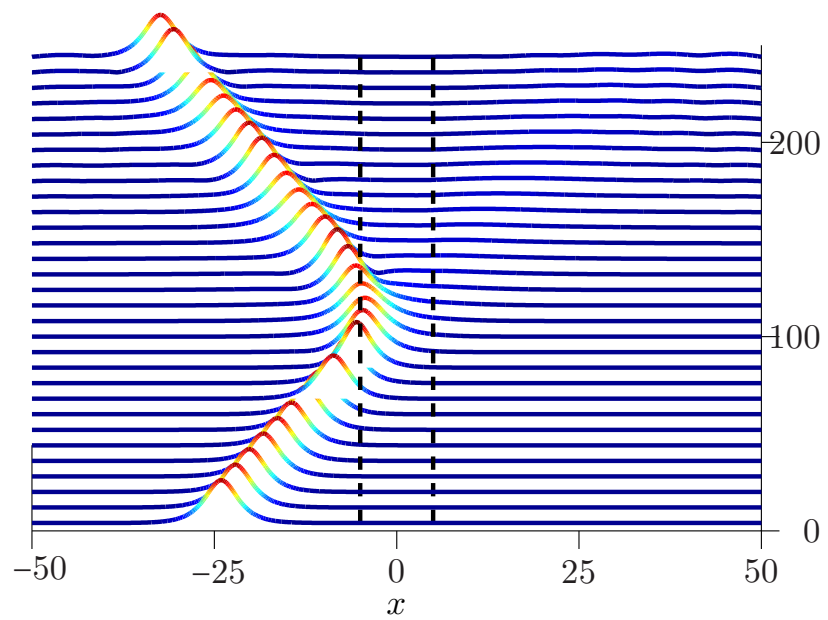

(a)

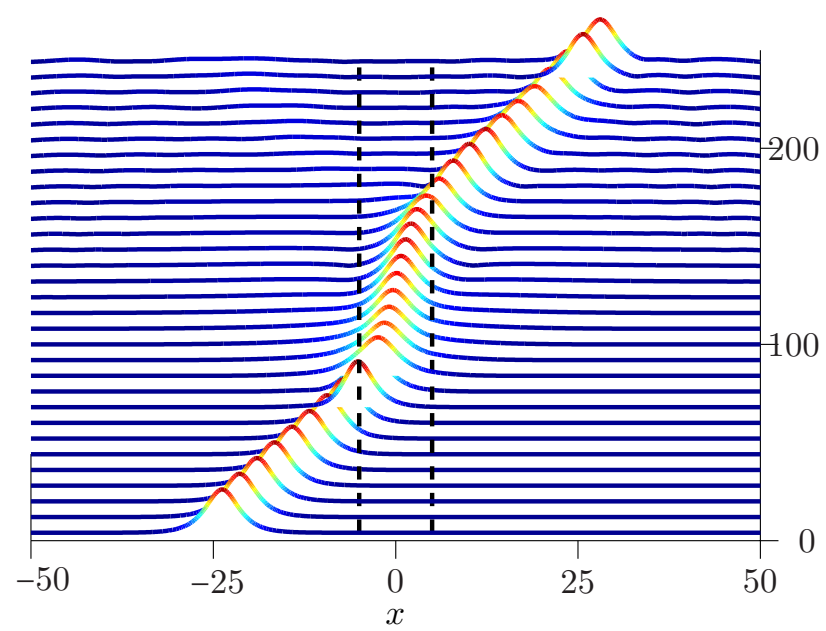

(c)

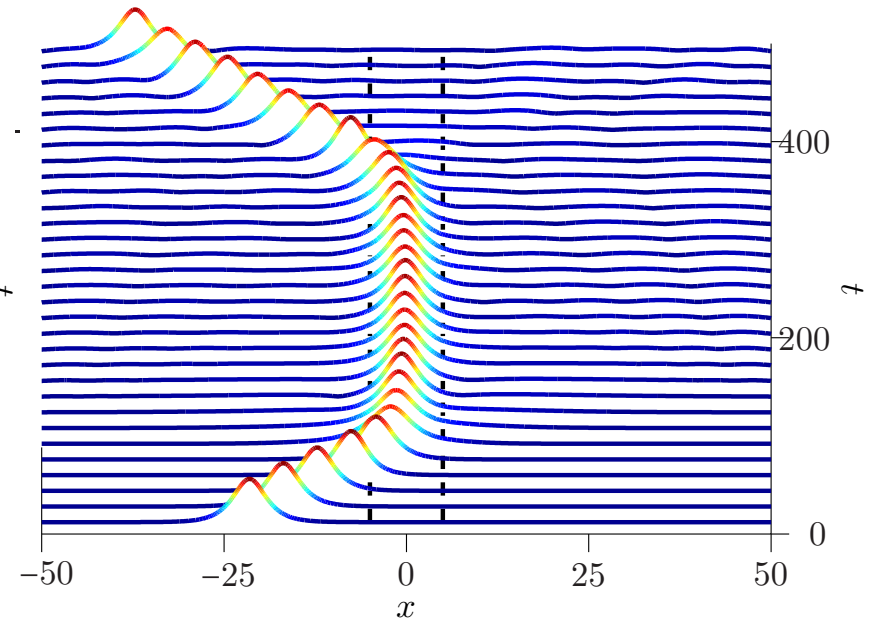

(b)

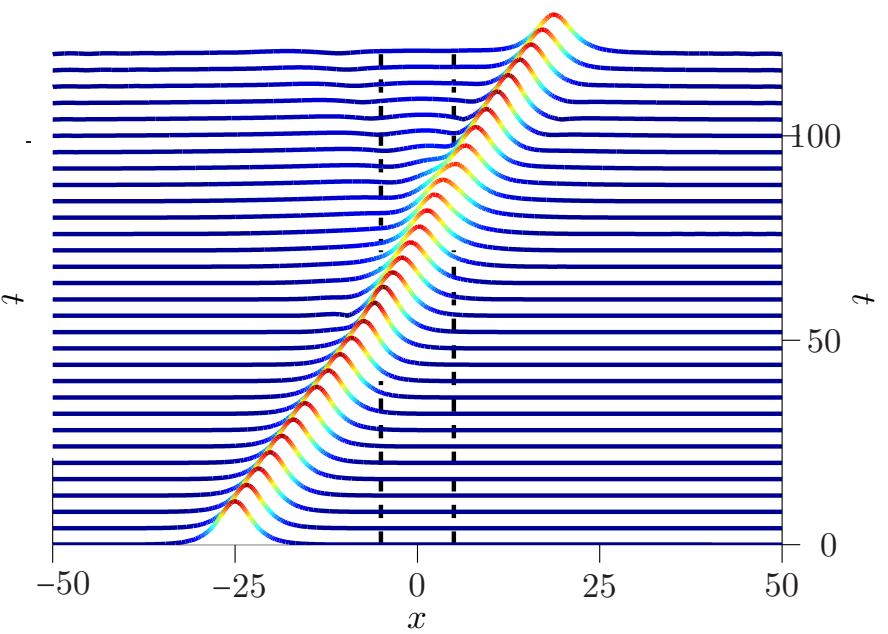

(d)

Figure 1. (Color online) Time evolution of the condensate density. The external potential, indicated by the dashed lines, is a square barrier with height $V_{0}=0.04$ and width $b=10$ and the incoming soliton has $N=1$. The threshold velocity, Equation (37), is $v_{\mathrm{t}} \approx 0.289$. In (a) the incoming soliton velocity is $v=0.24$, which is well below the threshold velocity, in (b) the velocity is $v=0.29$, which is just about the threshold velocity and in (c,d) the velocities are $v=0.3$ and $v=0.4$ respectively, which are above the threshold velocity. Here and in all the following figures all physical quantities are presented in the dimensionless units defined by Equations (3)-(6).

To get a better understanding of the observed phenomena, we assume that the soliton keeps its sech-shape during the scattering process, a fair assumption as seen from the numerical calculations in Figure 1,

$$
\psi_{x_{0}}(x)=B \operatorname{sech}\left[B\left(x-x_{0}\right)\right] \mathrm{e}^{i \dot{x}_{0}\left(x-x_{0}\right)+i \phi},
$$

and we use the Lagrange approach presented in $[17,18,42]$ to identify equations of motion for the time dependent parameters $B, x_{0}$ and $\phi$. The effective Lagrangian of the system is

$$
\mathcal{L}=\frac{1}{2} N \dot{x}_{0}^{2}-N \dot{\phi}+\frac{1}{6} N B(N-B)-V_{\text {eff }}\left(x_{0}\right),
$$

where $V_{\text {eff }}\left(x_{0}\right)$ is

$$
V_{\text {eff }}\left(x_{0}\right)=\int_{-\infty}^{\infty} \mathrm{d} x V(x)\left|\psi_{x_{0}}(x)\right|^{2}
$$


For a square barrier, i.e., an external potential of the form

$$
V(x)= \begin{cases}0 & \text { for }|x|>\frac{b}{2} \\ V_{0} & \text { for }|x|<\frac{b}{2}\end{cases}
$$

the effective potential becomes

$$
V_{\text {eff }}\left(x_{0}\right)= \begin{cases}0 & \text { for }|x|>\frac{b}{2} \\ \frac{V_{0} N}{2}\left(\tanh \left[B\left(\frac{b}{2}-x_{0}\right)\right]\right. & \\ \left.+\tanh \left[B\left(\frac{b}{2}+x_{0}\right)\right]\right) & \text { for }|x|<\frac{b}{2}\end{cases}
$$

The Hamiltonian of the motion is found from the Lagrangian:

$$
H=N \dot{x}_{0}^{2}-\mathcal{L}=\frac{1}{2} N \dot{x}_{0}^{2}+\frac{1}{6} N B(B-N)+V_{\text {eff }}\left(x_{0}\right),
$$

and from this we find the classical equation of motion for the soliton center $x_{0}$

$$
\ddot{x}_{0}=-\frac{1}{N} \frac{\partial V_{\text {eff }}\left(x_{0}\right)}{\partial x_{0}} .
$$

This is the equation of motion of a classical particle with mass $N$ and center of mass $x_{0}$ moving in the effective potential, $V_{\text {eff }}$, defined by Equation (32). This, as well as particle number conservation and the variation of the soliton width, can also be found from the Lagrangian equations.

The threshold incident energy needed by the soliton to cross the barrier is given by

$$
\frac{1}{2} N v_{\mathrm{t}}^{2}-\frac{N^{3}}{24}=\max _{\left|x_{0}\right|<\frac{b}{2}}\left\{\frac{1}{6} N B(B-N)+V_{\text {eff }}\left(x_{0}\right)\right\} .
$$

The corresponding threshold velocity, $v_{\mathrm{t}}$, of the bright soliton is indicated by the dashed lines in Figure 2, and reproduces the transition between complete reflection and transmission by the potential.

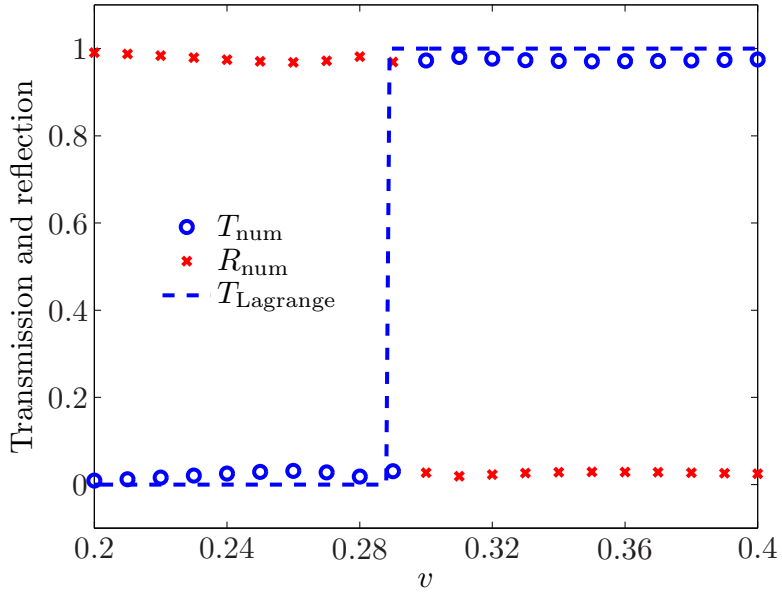

(a)

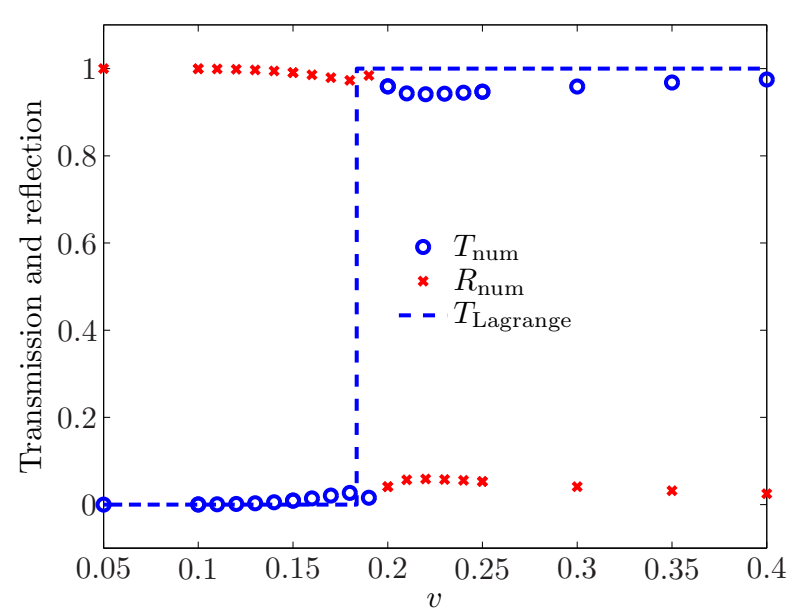

(b)

Figure 2. (Color online) Transmission and reflection coefficients of a soliton scattering on a weak square barrier for various soliton velocities found by a numerical solution of the GPE (symbols) and by the Lagrangian method (dashed lines). The soliton has $N=1$ and the barrier height is $V_{0}=0.04$. In (a) the barrier has width $b=10$, and is thus wide compared to the soliton width $B^{-1}=2$. In (b) the barrier has width $b=2$ which is comparable to the soliton width. 
We also note that since the barrier is softened by the interparticle interactions, Equation (34), it makes sense that the soliton penetrates into the classical forbidden areas as seen in Figure 1 [16]. The results presented here are similar to the ones presented in $[27,28]$ while scattering of solitons and breathers on delta-function barriers tend to transmit or reflect all atoms populating the individual solitonic waves [38,39].

\subsection{Strong Barriers}

Scattering of bright solitons on strong barriers with $V_{0} \gg \mu$ has been studied in the limit of high soliton velocities $[18,19,23,24]$. In this regime the soliton wave function may coherently split into reflected and transmitted components, and the asymptotic behavior of the transmission and reflection as the soliton velocity increases is the same as for a single particle, described by the linear Schrödinger equation. In the limit of low soliton velocities the soliton is also split into a reflected and a transmitted component. Here, we shall consider scattering of bright solitons on strong potentials with special attention to the behavior at low soliton velocities, where the single particle description fails.

Figure 3 shows the time evolution of a bright soliton incident on a rectangular potential barrier. We observe both a reflected and a transmitted component in the calculation. When the potential barrier is high enough to split the soliton, i.e., $V_{0} \gg \mu$, and at the same time wide compared to the soliton width, a part of the soliton is seen to be transiently trapped inside the barrier, see Figure 4. The trapped component oscillates and is gradually emitted out of the barrier region. Numerical simulations as well as analytical calculations show that this trapping is due to the wave nature of the soliton, and not the interactions. This behavior is not seen when the barrier is narrow compared to the soliton width.

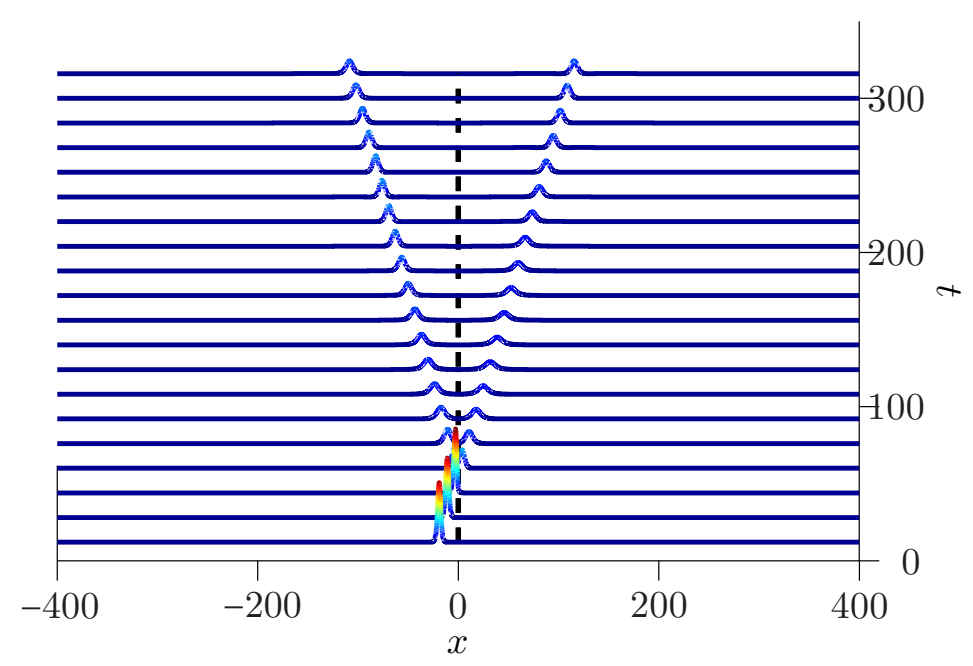

Figure 3. (Color online) Time evolution of the condensate density. The external potential is a square barrier placed at $x=0$. The potential has height $V_{0}=0.8$ and width $b=0.5$, and the incoming soliton has $v=0.5$ and $N=1$. 


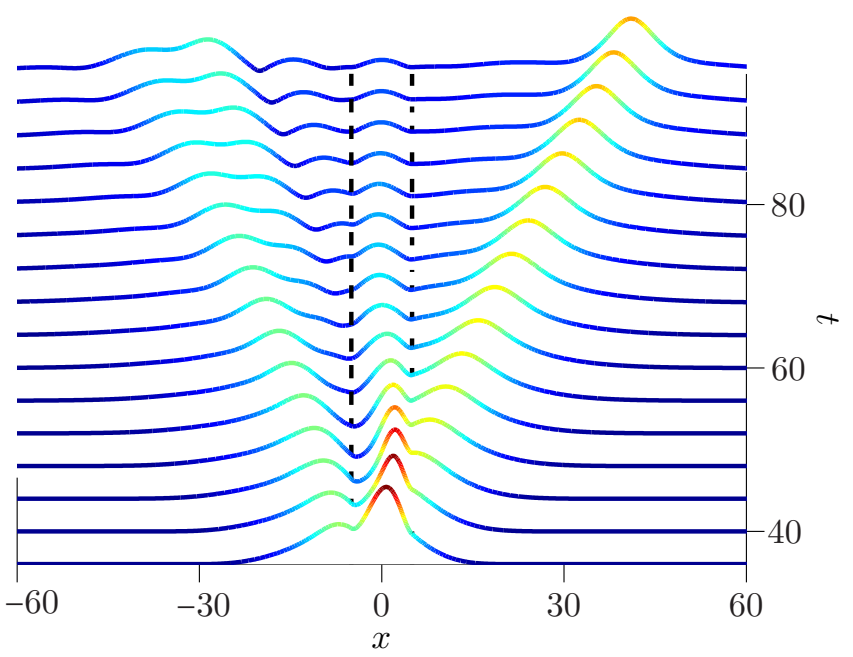

Figure 4. (Color online) Time evolution of the condensate wave function. The external potential is a square barrier placed at $x=0$. The potential has $V_{0}=0.2$ and $b=10$, and the incoming soliton velocity is $v=0.7$. Here we have zoomed in on the time evolution of the wave function $|\psi(x)|$ (not the density), in order to present the details better.

We have solved the GPE numerically for different cases, and Figure 5 summarizes our results (symbols) as functions of the incident velocity. When the external potential is strong compared to the chemical potential of the soliton, we expect the interaction term in the GPE to be less important. The results in Figure 5 may thus be approximated by the usual expression for the transmission probability for a single particle with energy $E$ incident on a square potential barrier,

$$
\begin{aligned}
T=\Theta & (E) \times \\
& \left\{\begin{array}{r}
{\left[1+\frac{V_{0}^{2}}{4 E\left(V_{0}-E\right)} \sinh ^{2}\left(b \sqrt{2\left(V_{0}-E\right)}\right)\right]^{-1}} \\
\text { for } E<V_{0} \\
{\left[1+\frac{V_{0}^{2}}{4 E\left(E-V_{0}\right)} \sinh ^{2}\left(b \sqrt{2\left(E-V_{0}\right)}\right)\right]^{-1}} \\
\text { for } E>V_{0},
\end{array}\right.
\end{aligned}
$$

where the Heaviside step function, $\Theta(E)$, ensures that the energy of the particle scattered is positive, which is evident in the single particle case but not in the interacting particle case. The non-linear interaction term cannot be omitted from the GPE in regions of space away from the strong potential barrier. In these regions, however, the soliton behavior is well known, and in particular we know the average energy per particle $\epsilon$ :

$$
\epsilon=\frac{E_{\mathrm{B}}}{N}=\frac{1}{2} v^{2}-\frac{N^{2}}{24}
$$

Because of the attractive interactions between the particles, $\epsilon$ becomes negative when $v<\frac{N}{\sqrt{12}}$ (we have chosen $N=1$ in our calculations which gives $\frac{N}{\sqrt{12}} \approx 0.29$ in our dimensionless units).

To describe scattering of the soliton in the linear approximation, it makes good sense to replace the energy $E$ in Equation (38) by the average energy per particle in the soliton, $\epsilon$ in Equation (39), and in the velocity regime $v \leq \frac{N}{\sqrt{12}}$ the Heaviside stepfunction in Equation (38) suppresses the transmission and predicts full reflection of the soliton. The prediction based on this description is shown in Figure 5 and is in very good agreement with the solution of the GPE. The small transmission probability observed below the velocity threshold may be due, in parts, to our use of wave packets with finite spread, and hence with a momentum distribution exceeding the threshold value. 


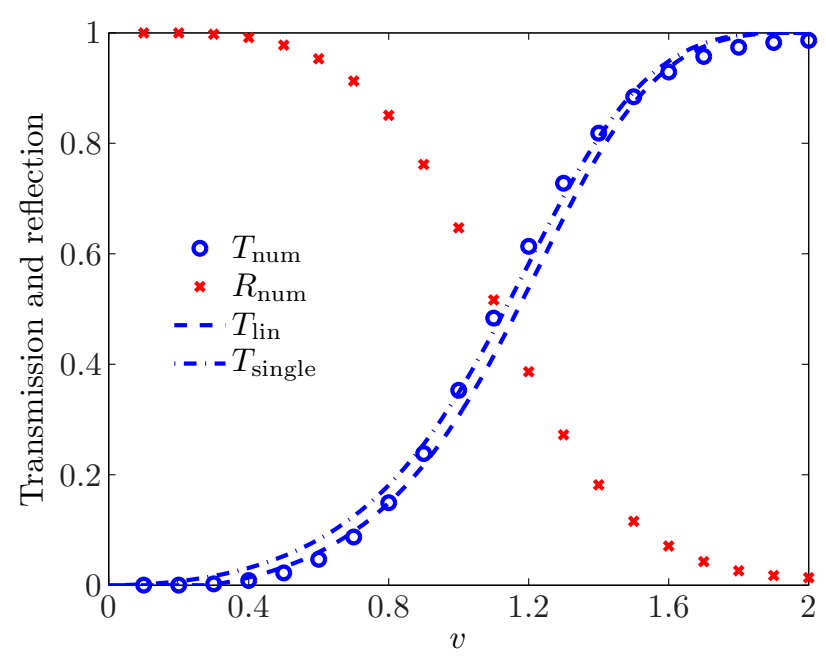

(a)

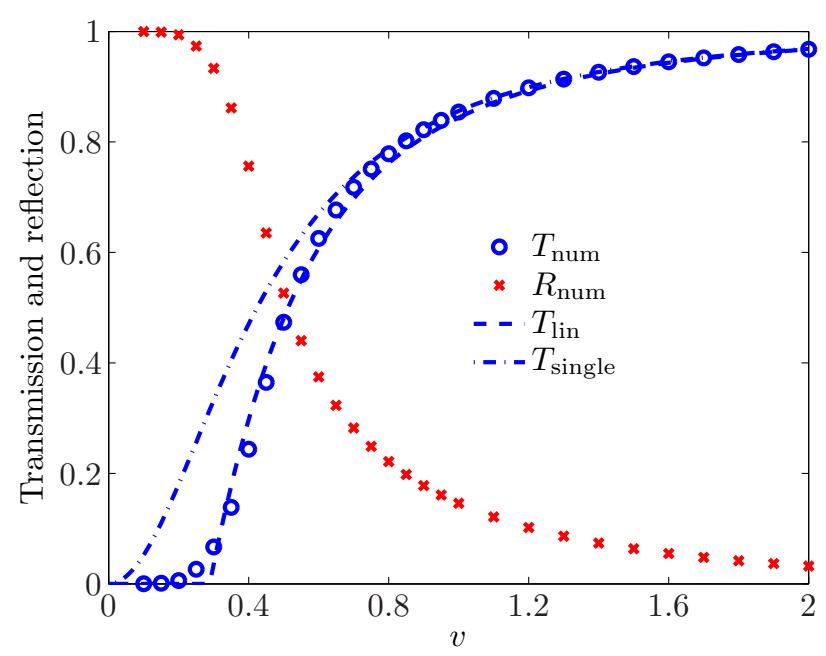

(c)

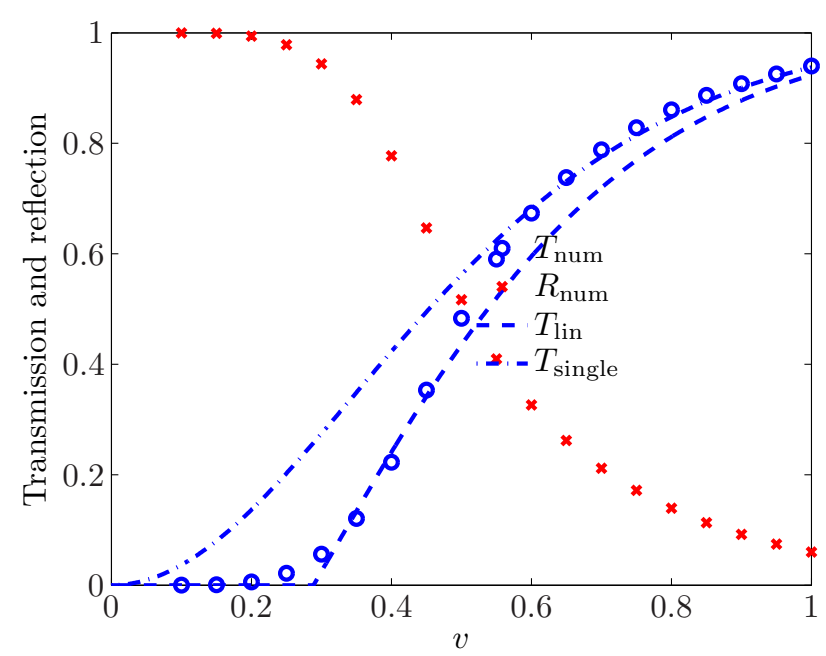

(b)

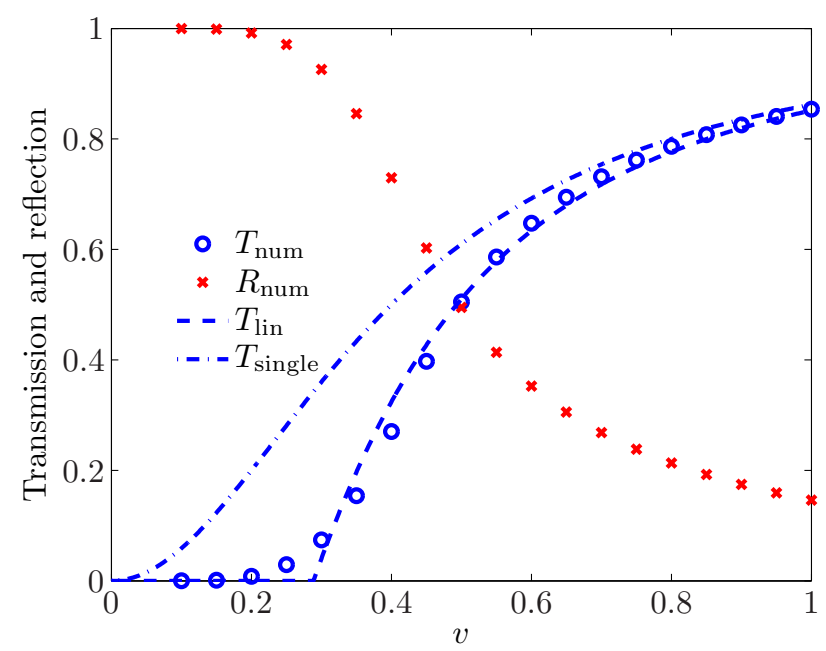

(d)

Figure 5. (Color online) Transmission and reflection coefficients of a soliton with $N=1$ on a strong barrier for various incoming soliton velocities. The coefficients have been found by solving the GPE numerically (symbols), by Equation (38) with $E=\frac{1}{2} v^{2}$ (dashed-dotted lines), and from Equation (38) with $E=\frac{1}{2} v^{2}-\frac{N^{2}}{24}$ (dashed lines), see the text. In (a) the barrier has strength $V_{0}=0.6$ and width $b=2$, in (b) the barrier has strength $V_{0}=0.2$ and strength $b=2$, and in (c) the barrier has $V_{0}=0.8$ and $b=0.5$, and thus the same area as in (b). All barriers are higher than the chemical potential, $|\mu|=\frac{1}{8}$. In (d) the barrier is a delta barrier of strength $\alpha=0.4$ which means that the barrier has the same area as the barriers in $(\mathbf{b}, \mathbf{c})$. For the delta potential the transmission probability reads $T=\Theta(E) \cdot \frac{1}{1+a^{2}}, a=\frac{\alpha}{\sqrt{2 E}}$.

\section{Scattering of Dark-Bright Solitons}

Dark and dark-bright solitons retain their localized nature when they collide or propagate in slowly varying potentials [4-6,41,45-48], and their scattering on delta-function potentials has been studied in [49]. Here, we shall study the collisions of dark-bright vector 
solitons on a rapidly varying square potential, which we choose to only directly affect the bright component such that the coupled GP equations are given by

$$
\begin{aligned}
i \frac{\partial \psi_{\mathrm{D}}(x, t)}{\partial t} & =\left(-\frac{1}{2} \frac{\partial^{2}}{\partial x^{2}}+\left|\psi_{\mathrm{D}}(x, t)\right|^{2}\right. \\
& \left.+\left|\psi_{\mathrm{B}}(x, t)\right|^{2}\right) \psi_{\mathrm{D}}(x, t), \\
i \frac{\partial \psi_{\mathrm{B}}(x, t)}{\partial t} & =\left(-\frac{1}{2} \frac{\partial^{2}}{\partial x^{2}}+\left|\psi_{\mathrm{B}}(x, t)\right|^{2}\right. \\
& \left.+\left|\psi_{\mathrm{D}}(x, t)\right|^{2}+V(x)\right) \psi_{\mathrm{B}}(x, t) .
\end{aligned}
$$

Because of the rapid spatial variation at the edge of the square potential, we expect the vector soliton to break up into a reflected and a transmitted part as we observed for the single bright soliton. However due to the interaction with the dark component, this system is more complicated, and the linear approximation successfully applied to the bright soliton is not valid here.

The results, shown in Figure 6, are obtained by numerical simulations of the system (in all numerical calculations $\mu=1$ ). It is seen that even though the external potential only affects the bright component directly, the dark component feels the external potential through its interaction with the bright component. Both components of the dark-bright soliton are partly reflected and partly transmitted through the external barrier. After impact on the external potential the reflected(transmitted) bright and dark components are still bound in joint center of mass motion.

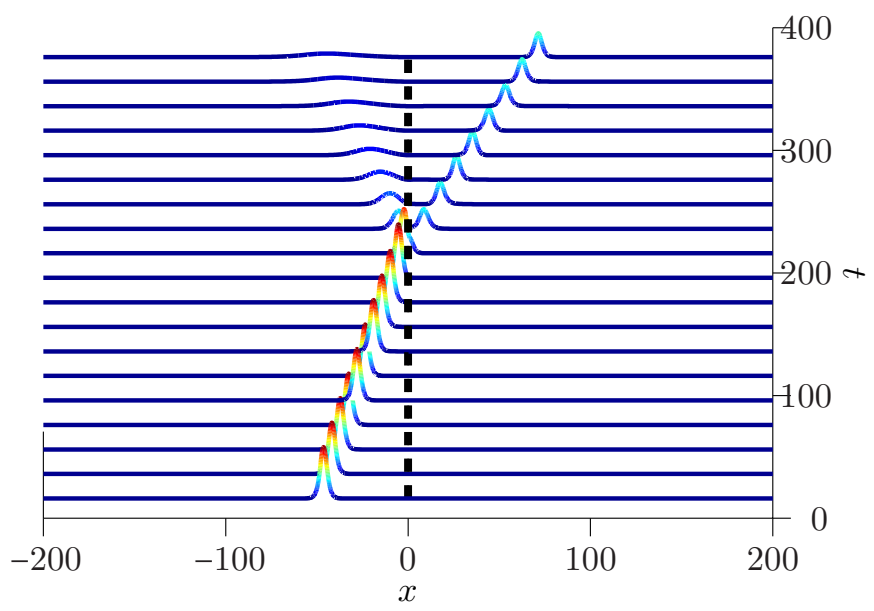

(a)

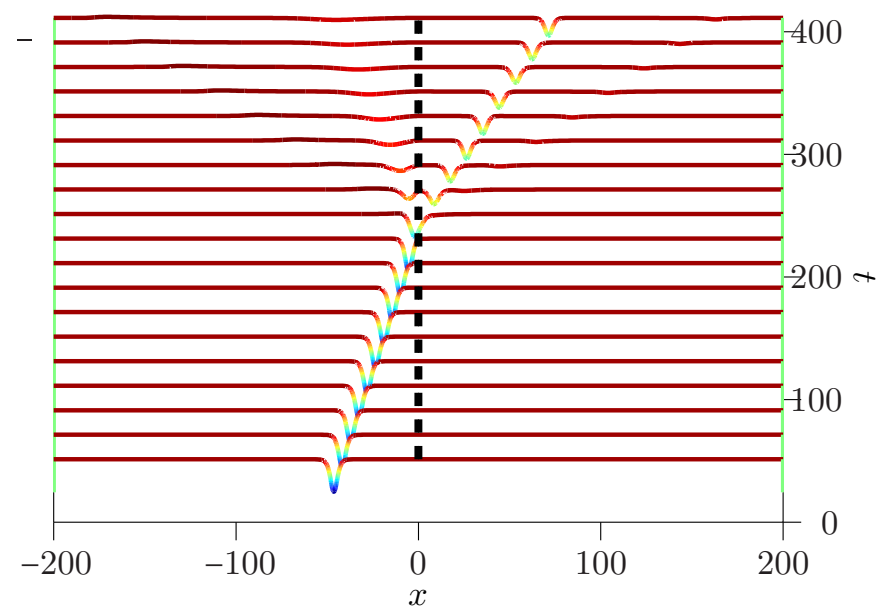

(b)

Figure 6. (Color online) Time evolution of the condensate density when the dark-bright soliton scatters on a barrier. (a) shows the bright component and (b) the dark component of the condensate. The incoming soliton has $N_{\mathrm{B}}=3$ and $\sin \alpha=0.5$, and the barrier, which only directly affects the bright component, has height $V_{0}=0.2$ and width $b=2$, its location is indicated by the dashed lines.

The transmission and reflection coefficients, $T_{\mathrm{B}}$ and $R_{\mathrm{B}}$, are defined in the same way as for the single bright soliton in Equation (29). For the dark component, we define the reflection and transmission coefficients as the number of atoms missing with respect to the homogeneous background condensate, on the reflection and transmission side of the 
barrier, divided by the missing number of atoms $N_{D}$ in the incident dark component of the vector soliton,

$$
\begin{aligned}
& T_{\mathrm{D}}=\frac{1}{N_{\mathrm{D}}} \int_{0}^{x_{\mathrm{sw}, T}} \mathrm{~d} x\left(\mu-\left|\psi_{\mathrm{D}}\right|^{2}\right), \\
& R_{\mathrm{D}}=\frac{1}{N_{\mathrm{D}}} \int_{x_{\mathrm{sw}, R}}^{0} \mathrm{~d} x\left(\mu-\left|\psi_{\mathrm{D}}\right|^{2}\right) .
\end{aligned}
$$

The limits $x_{\mathrm{sw}, T}$ and $x_{\mathrm{sw}, R}$ are determined such that the amplitude variations due to sound waves emitted into the $\psi_{\mathrm{D}}$-component during the scattering process do not contribute. We note from Figure 7 that both the bright and the dark soliton are indeed split by the potential. The dark soliton, however, is not reflected to the same degree as the bright one, and when the bright soliton reflection reaches unity, the dark soliton transmission converges to a constant value which is unchanged by any further increase of the barrier height, see Figure 7. The reflected bright component is, so to speak, only able to capture a certain amount of the dark one, and the weaker the bright component (smaller $N_{\mathrm{B}}$ ) the less of the dark component is asymptotically reflected. As was the case for the single bright soliton, the smaller of the transmitted and reflected component of the dark-bright soliton shows dispersion and is not completely solitonic. In Figure 7 it is also seen that the reflection coefficient and the transmission coefficient of the dark soliton do not add to one when the barrier height is increased. This we ascribe to sound waves forming wave packets that carry a corresponding surplus of atoms in the background condensate. It has been proposed to investigate the shape of a perturbing potential by analysis of the scattered Bogoliubov sound waves [50], and within the range of validity of Bogoliubov theory, one may obtain full tomographic information about the scattered quantum state [51].

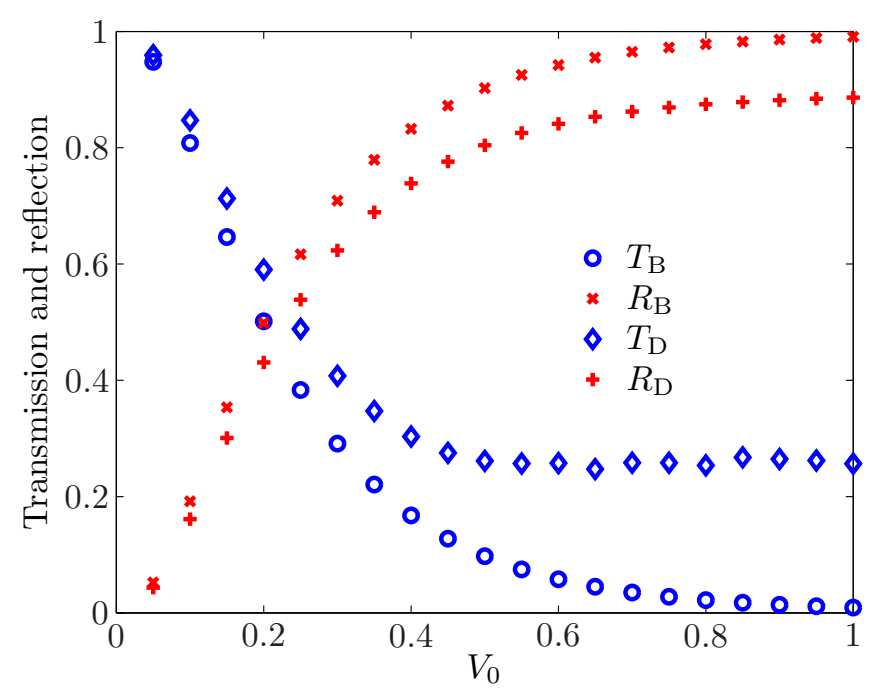

(a)

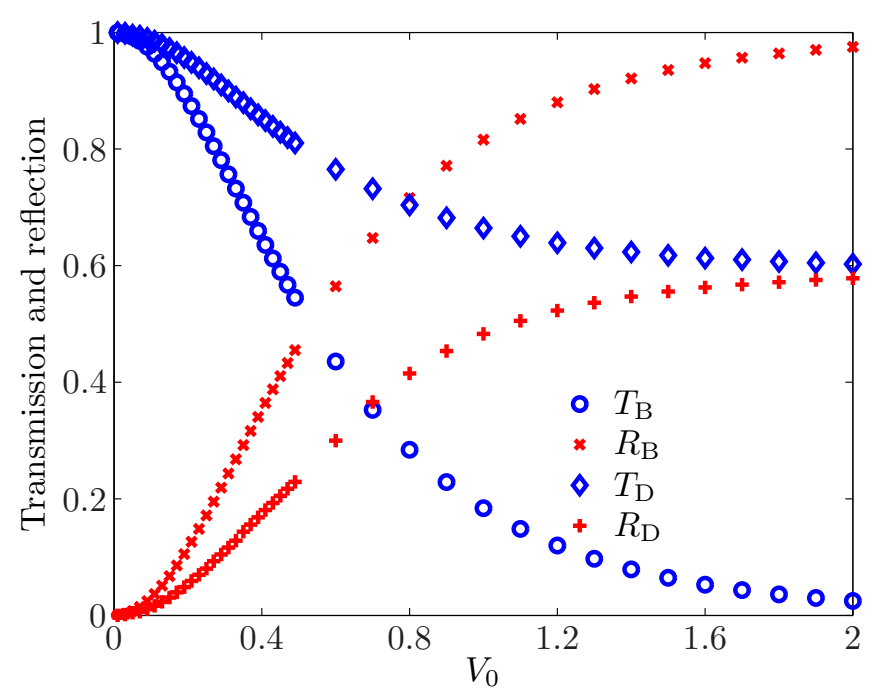

(b)

Figure 7. Transmission and reflection coefficients of the dark soliton and the bright soliton as a function of the barrier height $V_{0}$. In (a) $N_{\mathrm{B}}=3$ and in (b) $N_{\mathrm{B}}=1$. In both cases $\sin \alpha=0.5$ and the barrier width is that of a dark-bright soliton with $\sin \alpha=0\left(\kappa^{-1}\right.$, Equation (22)), for $N_{\mathrm{B}}=3$ that is $b=2$ and for $N_{\mathrm{B}}=1$ it is $b \approx 1.3$.

\section{Conclusions}

We have investigated scattering of solitons in Bose-Einstein condensates on localized potentials. The homogeneous Gross-Pitaevskii equation supports matter wave solitons, and we have focused on two types of solitons: Bright solitons, which are wave packets without dispersion due to the attractive, non-linear interactions between the atoms in the soliton, 
and dark-bright solitons in two separate condensate components. In the latter the bright soliton is stabilized by the presence of the dark soliton, a dip in the condensate density.

The scattering properties of a dark-bright soliton on a barrier only affecting the bright component could be expected to resemble that of a single bright soliton scattering on a barrier. But we find that the scattering of a dark-bright soliton is in fact very different. The differences are due to the interaction between the two components of the dark-bright condensate, which enables an indirect interaction between the dark soliton and the external potential. This interaction is strongest when the bright component is large compared to the dark one.

We have obtained numerical results by mean field theory and provided their interpretation by classical particle and single particle wave function arguments. The splitting of solitons by scattering potentials has also been proposed as a promising candidate for observation of effects beyond Gross-Pitaevskii mean field theory, thus exploring the atomic equivalent to non-classical states in quantum optics. Scattering with partial reflection on a potential barrier may thus produce Schrödinger cat like, mesoscopic superposition states with all atoms in the reflected and in the transmitted wavepacket mode [22,29]. Such states as well as states with other types of occupation number correlations have promising applications in metrology $[30,53,54,56]$, but they are fragile against disintegration of the state and spreading of the atoms over many single particle states. A proper analysis of such effects thus necessitate multi-atom, multi-mode theory like the multi-orbital theory [55], positive-P simulations $[28,37,56]$ or truncated Wigner approaches $[28,57]$. The work presented here may guide efforts and assess relevant parameter regimes prior to more advanced research efforts beyond mean field theory and single and few mode approximations. We note that experimental criteria have been proposed for when the atomic system may be restricted to populate only few scattering modes [58]. Experimental realization of solitons in 1Dcondensates is well developed [59], and dark-bright solitons have previously been studied in slowly varying potentials. Thus the prospects are promising for experimental studies along the lines of our calculations.

Author Contributions: Conceptualization, S.D.H., N.N. and K.M.; formal analysis, S.D.H., N.N. and K.M.; writing—original draft preparation, S.D.H., N.N. and K.M.; supervision, N.N. and K.M.; All authors have read and agreed to the published version of the manuscript.

Funding: APC was funded by Villum Fonden: QUSCOPE.

Institutional Review Board Statement: Not applicable.

Informed Consent Statement: Not applicable.

Data Availability Statement: Not applicable.

Conflicts of Interest: The authors declare no conflict of interest.

\section{References}

1. Kivshar, Y.S.; Luther-Davies, B. Dark optical solitons: Physics and applications. Phys. Rep. 1998, 298, 81-197. [CrossRef]

2. Agrawal, G.P. Nonlinear Fiber Optics; Academic Press: Cambridge, UK, 2007.

3. Amico, L.; Boshier, M.; Birkl, G.; Minguzzi, A.; Miniatura, C.; Kwek, L.-C.; Aghamalyan, D.; Ahufinger, V.; Andrei, N.; Arnold, A.S.; et al. Roadmap on atomtronics. arXiv 2020, arXiv:2008.04439.

4. Pethick, C.; Smith, H. Bose-Einstein Condensation in Dilute Gases; Cambridge University Press: Cambridge, UK 2008.

5. Becker, C.; Stellmer, S.; Soltan-Panahi, P.; Dörscher, S.; Baumert, M.; Richter, E.-M.; Kronjäger, J.; Bongs, K.; Sengstock, K. Oscillations and interactions of dark and dark-bright solitons in Bose-Einstein condensates. Nat. Phys. 2008, 4, 496-501. [CrossRef]

6. Middelkamp, S.; Chang, J.; Hamner, C.; Carretero-Gonzlez, R.; Kevrekidis, P.; Achilleos, V.; Frantzeskakis, D.; Schmelcher, P.; Engels, P. Dynamics of dark-bright solitons in cigar-shaped Bose-Einstein condensates. Phys. Lett. A 2011, 375, $642-646$. [CrossRef]

7. Hamner, C.; Chang, J.; Engels, P.; Hoefer, M. Generation of dark-bright soliton trains in superfluid-superfluid counterflow. Phys. Rev. Lett. 2011, 106, 065302. [CrossRef] [PubMed] 
8. Strecker, K.; Partridge, G.; Truscott, A.; Hulet, R. Formation and propagation of matter-wave soliton trains. Nature 2002, 417, 150-153. [CrossRef] [PubMed]

9. Al Khawaja, U.; Stoof, H.T.C.; Hulet, R.G.; Strecker, K.E.; Partridge, G.B. Bright soliton trains of trapped Bose-Einstein condensates. Phys. Rev. Lett. 2002, 89, 200404. [CrossRef]

10. Scharf, R.; Bishop, A.R. Soliton chaos in the nonlinear Schrodinger equation with spatially periodic perturbations. Phys. Rev. A 1992, 46, R2973. [CrossRef]

11. Scharf, R.; Bishop, A.R. Length-scale competition for the one-dimensional nonlinear Schrodinger equation with spatially periodic potentials. Phys. Rev. E 1993, 47, 1375. [CrossRef]

12. Busch, T.; Anglin, J.R. Motion of dark solitons in trapped Bose-Einstein condensates. Phys. Rev. Lett. 2000, 84, 2298. [CrossRef]

13. Burger, S.; Bongs, K.; Dettmer, S.; Ertmer, W.; Sengstock, K.; Sanpera, A.; Shlyapnikov, G.V.; Lewenstein, M. Dark solitons in Bose-Einstein condensates. Phys. Rev. Lett. 1999, 83, 5198. [CrossRef]

14. Reinhardt, W.P.; Clark, C.W. Soliton dynamics in the collisions of Bose-Einstein condensates: An analogue of the Josephson effect. J. Phys. B 1997, 30, L785. [CrossRef]

15. Denschlag, J.; Simsarian, J.; Feder, D.; Clark, C.; Collins, L.; Cubizolles, J.; Deng, L.; Hagley, E.; Helmerson, K.; Reinhardt, W.; et al. Generating solitons by phase engineering of a Bose-Einstein condensate. Science 2000, 287, 97-101. [CrossRef]

16. Nogami, Y.; Toyama, F. Nonlinear Schrodinger soliton impinging on a potential step. Phys. Lett. A 1994, 184, 245-250. [CrossRef]

17. Young-Shan, C.; Rong-Zhou, G.; Hong, L. Motion of a Bose-Einstein Condensate Bright Soliton Incident on a Step-Like Potential. Chin. Phys. Lett. 2007, 24, 35. [CrossRef]

18. Sakaguchi, H.; Tamura, M. Scattering and trapping of nonlinear Schrodinger solitons in external potentials. J. Phys. Soc. Jpn. 2004, 73, 503-506. [CrossRef]

19. Ernst, T.; Brand, J. Resonant trapping in the transport of a matter-wave soliton through a quantum well. Phys. Rev. A 2010, 81, 033614. [CrossRef]

20. Lee, C.; Brand, J. Enhanced quantum reflection of matter-wave solitons. Europhys. Lett. 2006, 73, 321. [CrossRef]

21. Miroshnichenko, A.; Flach, S.; Malomed, B. Resonant scattering of solitons. Chaos 2003, 13, 874. [CrossRef]

22. Weiss, C.; Castin, Y. Creation and Detection of a Mesoscopic Gas in a Nonlocal Quantum Superposition. Phys. Rev. Lett. 2009, 102, 010403. [CrossRef] [PubMed]

23. Holmer, J.; Marzuola, J.; Zworski, M. Soliton splitting by external delta potentials. J. Nonlinear Sci. 2007, 17, 349. [CrossRef]

24. Cao, X.; Malomed, B. Soliton-defect collisions in the nonlinear Schrödinger equation. Phys. Lett. A 1995, 206, 177. [CrossRef]

25. Goodman, R.; Holmes, P.; Weinstein, M. Strong NLS soliton-defect interactions. Phys. D 2004, 192, 215. [CrossRef]

26. Wang, C.-H.; Hong, T.-M.; Lee, R.-K.; Wang, D.-W. Particle-wave duality in quantum tunneling of a bright soliton. Opt. Express 2012, 20, 22675. [CrossRef] [PubMed]

27. Helm, J.L.; Billam, T.P.; Gardiner, S.A. Bright matter-wave soliton collisions at narrow barriers. Phys. Rev. A 2012, 85, 053621. [CrossRef]

28. Martin, A.D.; Ruostekoski, J. Quantum dynamics of atomic bright solitons under splitting and recollision, and implications for interferometry. New J. Phys. 2012, 14, 043040. [CrossRef]

29. Gertjerenken, B.; Billam, T.P.; Khaykovich, L.; Weiss, C. Quantum versus mean-field behavior. Phys. Rev. A 2012, 86033608. [CrossRef]

30. Gertjerenken, B.; Wiles, T.P.; Weiss, C. Progress towards quantum-enhanced interferometry with harmonically trapped quantum matter-wave bright solitons. Phys. Rev. A 2016, 94 053638. [CrossRef]

31. Grimshaw, C.L.; Gardiner, S.A.; Malomed, B.A. Splitting of two-component solitary waves from collisions with narrow potential barriers. Phys. Rev. A 2020, 101, 043623. [CrossRef]

32. Cuevas, J.; Kevrekidis, P.G.; Malomed, B.A.; Dyke, P.; Hulet, R.G. Interactions of solitons with a Gaussian barrier: Splitting and recombination in quasi-one-dimensional and three-dimensional settings. New. J. Phys. 2013, 15, 063006. [CrossRef]

33. Marchukov, O.V.; Malomed, B.A.; Dunjko, V.; Ruhl, J.; Olshanii, M.; Hulet, R.G.; Yurovsky, V.A. Quantum Fluctuations of the Center of Mass and Relative Parameters of Nonlinear Schrödinger Breathers. Phys. Rev. Lett. 2020, 125, 050405. [CrossRef] [PubMed]

34. Opnachuk, B.; Drummond, P.D. One-dimensional Bose gas dynamics: Breather relaxation. Phys. Rev. A 2017, 96, 053628. [CrossRef]

35. Luo, D.; Jin, Y.; Nguyen, J.H.V.; Malomed, B.A.; Marchukov, O.V.; Yurovsky, V.A.; Dunjko, V.; Olshanii, M.; Hulet, R.G. Creation and Characterization of Matter-Wave Breathers. Phys. Rev. Lett. 2020, 125, 183902. [CrossRef]

36. Yurovsky, V.A.; Malomed, B.A.; Hulet, R.G.; Olshanii, M. Dissociation of One-Dimensional Matter-Wave Breathers due to Quantum Many-Body Effects. Phys. Rev. Lett. 2017, 119, 220401. [CrossRef]

37. Ng, K.L.; Opanchuk, B.; Reid, M.D.; Drummond, P.D. Nonlocal Pair Correlations in a Higher-Order Bose Gas Soliton. Phys. Rev. Lett. 2019, 122, 203604. [CrossRef]

38. Marchukov, O.V.; Malomed, B.A.; Yurovsky, V.A.; Olshanii, M.; Dunjko, V.; Hulet, R.G. Splitting of nonlinear-Schrödingerequation breathers by linear and nonlinear localized potentials. Phys. Rev. A 2019, 99, 063623. [CrossRef]

39. Dunjko, V.; Olshanii, M. Resilience of constituent solitons in multisoliton scattering on barriers. arXiv 2020, arXiv:1501.00075v4.

40. Zaremba, E. Sound propagation in a cylindrical Bose-condensed gas. Phys. Rev. A 1998, 57, 518. [CrossRef] 
41. Busch, T.; Anglin, J.R. Dark-Bright Solitons in Inhomogeneous Bose-Einstein Condensates. Phys. Rev. Lett. 2001, 87, 010401. [CrossRef] [PubMed]

42. Sakaguchi, H.; Tamura, M. Scattering of Solitons and Dark Solitons by Potential Walls in the Nonlinear Schrödinger Equation. J. Phys. Soc. Jpn. 2005, 74, 292. [CrossRef]

43. Yue-Yue, W.; Jie-Fang, Z. Collision induced splitting of bright soliton in Bose-Einstein condensate. Chin. Phys. B 2009, 18, 1168 [CrossRef]

44. Cornish, S.; Parker, N.; Martin, A.; Judd, T.; Scott, R.; Fromhold, T.; Adams, C. Quantum reflection of bright matter-wave solitons. Phys. D Nonlinear Phenom. 2009, 238, 1299. [CrossRef]

45. Weller, A.; Ronzheimer, J.P.; Gross, C.; Esteve, J.; Oberthaler, M.K.; Frantzeskakis, D.J.; Theocharis, G.; Kevrekidis, P.G. Experimental Observation of Oscillating and Interacting Matter Wave Dark Solitons. Phys. Rev. Lett. 2008, 101, 130401. [CrossRef]

46. Kälbermann, G. Soliton interacting as a particle. Phys. Lett. A 1999, 252, 37. [CrossRef]

47. Liu, X.; Pu, H.; Xiong, B.; Liu, W.M.; Gong, J. Formation and transformation of vector solitons in two-species Bose-Einstein condensates with a tunable interaction. Phys. Rev. A 2009, 79, 013423. [CrossRef]

48. Nistazakis, H.E.; Frantzeskakis, D.J.; Kevrekidis, P.G.; Malomed, B.A.; Carretero-Gonzalez, R. Bright-dark soliton complexes in spinor Bose-Einstein condensates. Phys. Rev. A 2008, 77, 033612. [CrossRef]

49. Alotaibi, M.O.D.; Carr, L.D. Scattering of a dark-bright soliton by an impurity. J. Phys. B At. Mol. Opt. Phys. $2019,52,165301$. [CrossRef]

50. Marchukov, O.V.; Volosniev, A. Shape of a sound wave in a weakly-perturbed Bose gas. SciPost Phys. 2021, 10, 025. [CrossRef]

51. Mouritzen, A.S.; Mølmer, K.M. Tomographic reconstruction of quantum correlations in excited Bose-Einstein condensates. Phys. Rev. A 2005, 71, 033602. [CrossRef]

52. Poulsen, U.V.; Mølmer, K.M. Quantum beam splitter for atoms. Phys. Rev. A 2002, 65, 033613. [CrossRef]

53. Negretti, A.; Henkel, C.; Mølmer, K.M. Quantum-limited position measurements of a dark matter-wave soliton. Phys. Rev. A 2008, 77, 043606. [CrossRef]

54. Negretti, A.; Henkel, C.; Mølmer, K.M. Quantum fluctuations in the image of a Bose gas. Phys. Rev. A 2008, 78, 023630. [CrossRef]

55. Streltsov, A.I.; Alon, O.E.; Cederbaum, L.S. Scattering of an attractive Bose-Einstein condensate from a barrier: Formation of quantum superposition states. Phys. Rev. A 2009, 80, 043616. [CrossRef]

56. Poulsen, U.V.; Mølmer, K. Positive-P simulations of spin squeezing in a two-component Bose condensate. Phys. Rev. A 2001, 64, 013616. [CrossRef]

57. Laudat, T.; Dugrain, V.; Mazzoni, T.; Huang, M.-Z.; Alzar, C.L.G.; Sinatra, A.; Rosenbusch, P.; Reichel, J. Spontaneous spin squeezing in a rubidium BEC. New J. Phys. 2018, 20, 073018. [CrossRef]

58. Helm, J.L.; Rooney, S.J.; Gardiner, S.A. Splitting bright matter-wave solitons on narrow potential barriers: Quantum to classical transition and applications to interferometry. Phys. Rev. A 2014, 89, 033610. [CrossRef]

59. Garrett, M.C.; Ratnapala, A.; van Ooijen, E.D.; Vale, C.J.; Weegink, K.; Schnelle, S.K.; Vainio, O.; Heckenberg, N.R.; RubinszteinDunlop, H.; Davis, M.J. Growth dynamics of a Bose-Einstein condensate in a dimple trap without cooling. Phys. Rev. A 2011, 83, 013630. [CrossRef] 